\title{
Definitive single-stage posterior surgical correction of complete traumatic spondyloptosis at the thoracolumbar junction
}

\author{
Lee Sandquist, DO, Alexander Paris, BS, and Daniel K. Fahim, MD \\ Department of Neurosurgery, Oakland University William Beaumont School of Medicine, Royal Oak, Michigan
}

Complete dislocation at the thoracolumbar junction is a rare occurrence, with only 4 previously reported cases in 3 separate series. Surgical procedures in the reported cases of spondyloptosis at the thoracolumbar junction have been described using instrumentation, reduction, decompression, and stabilization techniques. In this report the authors' patient presented with spondyloptosis at the thoracolumbar junction, resulting in a T-11 American Spinal Injury Association Grade A injury. The authors corrected the patient's thoracolumbar spondyloptosis with surgical reconstruction without the use of leveraged instrumented reduction. They describe a single-stage, posterior-only spinal realignment, reconstruction, and stabilization. Within months of beginning postoperative therapy, the patient enrolled and attended courses at a local college and regained personal independence by learning to drive a motor vehicle with a hand control. Two-year radiographic and clinical follow-up confirms solid fusion across the reconstruction.

http://thejns.org/doi/abs/10.3171/2014.10.SPINE14165

KEY WORDS traumatic spondyloptosis; long-segment thoracolumbar instrumentation; traumatic spinal cord injury; thoracolumbar junction reconstruction; trauma

$\mathrm{T}$ RAUMATIC spondyloptosis is a rare spinal column injury resulting in $100 \%$ subluxation of a vertebral body most commonly in the sagittal plane. Highenergy injuries, such as motor vehicle accidents or falls from great heights, are the most frequent mechanisms of injury. ${ }^{2}$ Most reports of spondyloptosis describe fractures occurring at the lumbosacral junction or in the cervical spine. Complete dislocation at the thoracolumbar junction is a rare occurrence, with only 4 previously described cases in 3 series (Table 1). All resulted in American Spinal Injury Association (ASIA) Grade A spinal cord injuries (SCIs). Surgical procedures in the reported cases of spondyloptosis at the thoracolumbar junction have been described using instrumentation, reduction, decompression, and stabilization techniques..$^{1,9,10}$ Distraction techniques using leverage on the instrumentation were used to reduce the fractures and short-segment instrumented constructs with anterior support were used to reconstruct the thoracolumbar junction in all cases.

Here, we report a case of thoracolumbar spondyloptosis with surgical reconstruction without the use of leveraged instrumented reduction. We describe a single-stage, pos- terior-only spinal realignment, reconstruction, and stabilization procedure. This procedure involved a complete L-1 vertebrectomy to facilitate spinal column realignment without significant manipulation of the spinal column, thereby preventing potentially fatal manipulation of the vascular structures immediately anterior to the spinal column. Furthermore, the vertebrectomy provided ample autograft for optimization of fusion outcomes. A long-segment instrumented construct was performed using $\mathrm{O}$-arm navigation.

\section{Case Report}

History and Examination

A 20-year-old man without any previous medical history (except for the use of alcohol and marijuana) was involved in a low-speed motorcycle accident and presented with complete paraplegia, loss of rectal tone, a T-11 sensory level, and a prominent step off at the thoracolumbar junction. Therefore, he sustained a T-11 ASIA Grade A SCI. CT scanning and MRI of the entire spine were performed and revealed complete spondyloptosis at the thoracolumbar junction with a split fracture of the L-1 vertebral 
TABLE 1. Previous reports of cases of complete dislocation at the thoracolumbar junction*

\begin{tabular}{|c|c|c|c|c|c|c|}
\hline $\begin{array}{l}\text { Authors \& Year } \\
\text { (no. of patients) }\end{array}$ & Age (yrs) & Mechanism of Injury & $\begin{array}{l}\text { ASIA } \\
\text { Grade }\end{array}$ & $\begin{array}{c}\text { Fracture } \\
\text { Level }\end{array}$ & Surgical Reconstruction & Functional Outcome \\
\hline \multirow[t]{6}{*}{ Yadla et al., 2008 (5) } & $17-44$ & & & & & \\
\hline & & MVC & A & T10-12 & T8-L1 instrumentation & No recovery \\
\hline & & Steel beam fell on patient & A & T11-12 & Thoracic instrumentation & No recovery \\
\hline & & MVC (motorcycle) & C & L1-2 & T10-L4 instrumentation & Recovered ambulation \\
\hline & & Roof collapsed & A & T12-L1 & T10-L3 instrumentation & No recovery \\
\hline & & MVC (ejected) & $A$ & T7-8 & T3-11 instrumentation & No recovery \\
\hline \multirow[t]{5}{*}{$\begin{array}{l}\text { Chandrashekhara et al., } \\
2011 \text { (4) }\end{array}$} & $10-27$ & & & & & \\
\hline & & Run over by tractor & A & L3-4 & L2-5 instrumentation & No recovery \\
\hline & & MVC & A & L3-4 & L1-5 instrumentation & No recovery \\
\hline & & Fall from height & A & T12-L1 & T11-12 instrumentation & No recovery \\
\hline & & Fall from height & A & T12-L1 & T10-L2 instrumentation & No recovery \\
\hline \multirow[t]{3}{*}{ Sekhon et al., 2007 (2) } & $22 \& 36$ & & & & & \\
\hline & & $\begin{array}{l}\text { Large bale of hay fell on } \\
\text { neck \& shoulders }\end{array}$ & A & T6-7 & T5-8 instrumentation & No recovery \\
\hline & & $\begin{array}{l}\text { High-impact collision w/ } \\
\text { ceiling in a coal mine }\end{array}$ & A & T12-L1 & T11-12 instrumentation & No recovery \\
\hline
\end{tabular}

MVC = motor vehicle collision.

* Cases in boldface represent injuries sustained at the thoracolumbar junction.

body (Fig. 1). The patient did not have signs or symptoms of associated vascular injury.

\section{Operation}

A single-stage posterior fracture reduction and recon- structive surgery was performed. Upon completing our exposure, the entire L-1 vertebral body could be seen, resting behind the inferior aspect of the thoracic spine (Fig. 2A). The lacerated thecal sac was retracted inferiorly in the posteriorly displaced spinal canal corresponding
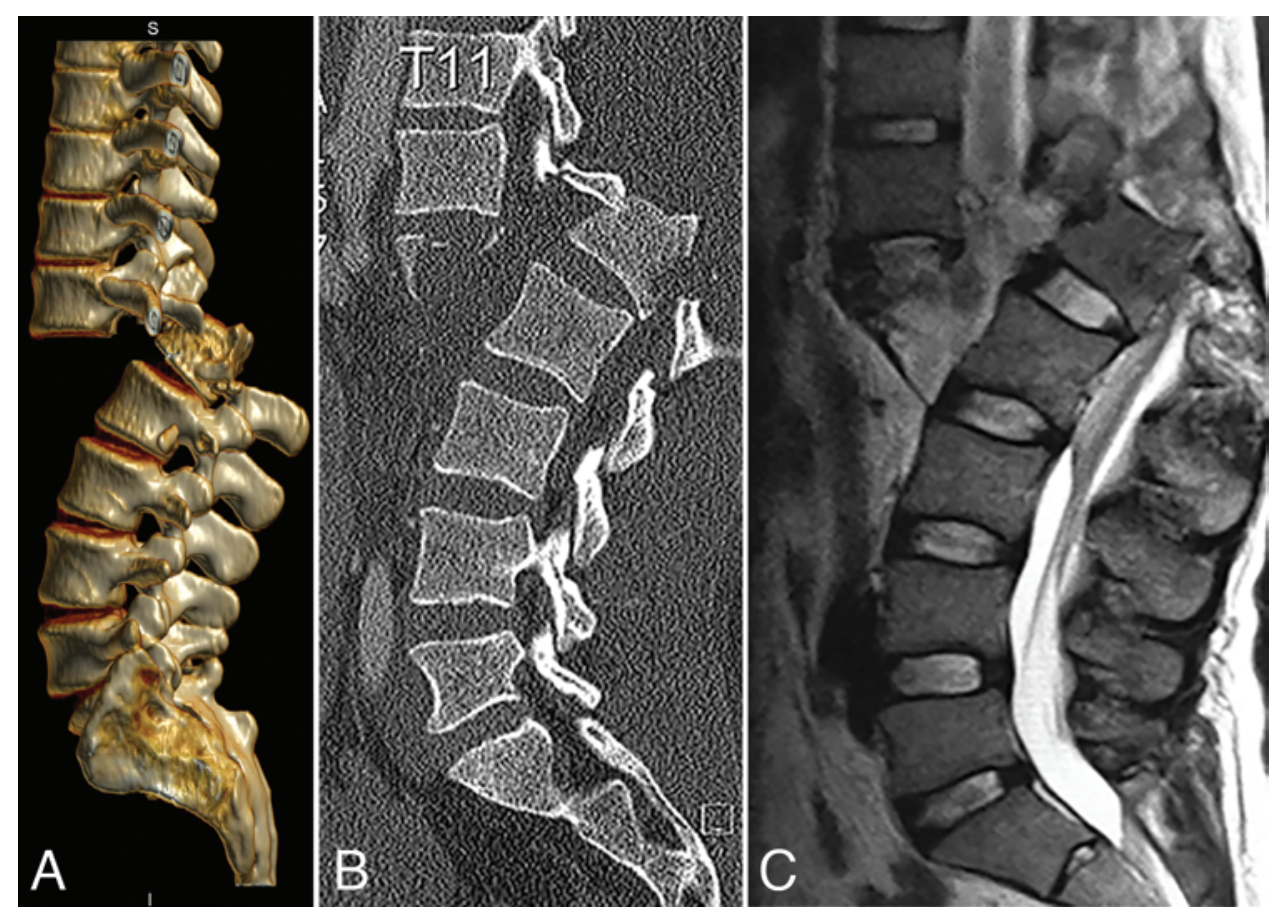

FIG. 1. A: Preoperative sagittal CT 3D reconstruction demonstrating the degree of bone injury. B: Preoperative sagittal CT scan demonstrating the degree of bone injury. C: Preoperative sagittal T2-weighted MR image of the thoracolumbar spine demonstrating the degree of injury to neural elements. 

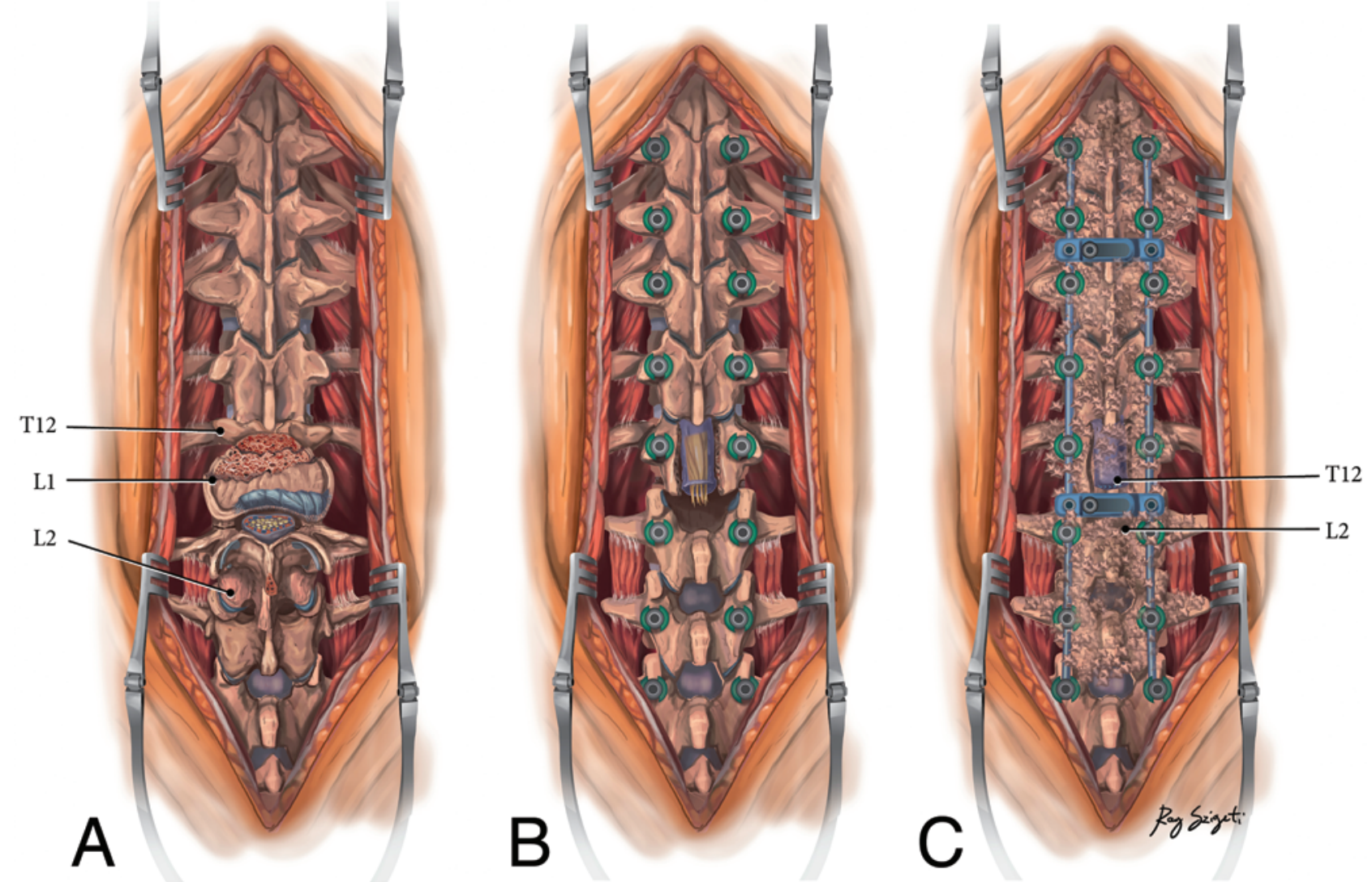

FIG. 2. Illustrations demonstrating preoperative lacerated dura and nerve roots (A), ligation of nerve roots and dura (B), and final repair of the dura with 6-0 Prolene suture and DuraSeal (C). Copyright Raymond Szigeti. Published with permission.

to the L-1 vertebral body. A portion of the T12-L1 disc remained along the posterior aspect of the L-1 superior endplate. The anterosuperior portion of the L-1 vertebral body endplate was fractured off and remained attached superiorly to the T12-L1 disc at the inferior extent of the thoracic spine (Fig. 2A).

The entire L-1 vertebral body was then removed, including all of the posterior elements. This provided ample autograft for our fusion. Because of the location of the L-1 vertebral body posterior to the inferior aspect of the thoracic spine, we did not have to be concerned about protecting any vascular structures during our vertebrectomy. The vascular structures were undisturbed in their position anterior to the thoracic spine. The L1-2 disc was also removed to allow for interbody fusion between L-2 and T-12 after realigning the spine.

The complete L-1 vertebrectomy exposed the fractured T-12 vertebral body. We then performed a completion laminectomy at T-12 to expose the lacerated thecal sac and truncated nerve roots (Fig. 2B). Superior extension of the laminectomy allowed identification of intact dura and visualization of the inferior aspect of the destroyed neural tissue (conus medullaris). The exiting T-11 nerve roots remained intact and were allowed to remain in position exiting at the T11-12 foramen bilaterally. The T-12 nerve roots could not be identified intraoperatively, and all other distal nerves had retracted inferiorly and also could not be readily visualized. The thecal sac was then closed inferiorly with a running 6-0 Prolene suture in a watertight fashion (Fig. 2C). A layer of DuraSeal (Covidien) was applied to the dura closure at the completion of the case.

We then had a clear path through the spinal canal to remove what remained of the T12-L1 disc. The posterior portion of the disc had remained attached to the posteriorly displaced L-1 vertebral body, and this was removed at the time of our vertebrectomy. The remaining portion of the T12-L1 disc was then removed and the endplate was decorticated to allow for interbody fusion between T-12 and L-2 after realigning the spine.

Having completed the vertebrectomy at L-1, the lumbar spine naturally fell anteriorly into a natural anatomical position inferior to the thoracic spine with minimal to no manipulation. No distraction forces were necessary to realign the spine due to the ample space provided between the posteriorly displaced lumbar spine and the thoracic spine that remained in anatomical position.

An intraoperative CT scan was obtained to verify the final anatomical vertebral column alignment following the complete vertebrectomy at L-1. O-arm navigation was used for pedicle screw placement. The construct included pedicle screw placement at T8-12 bilaterally and L2-4 bilaterally (Fig. 2B). L-1 was not instrumented, as it was completely removed and used for autograft arthrodesis.

Complete discectomies had already been performed 

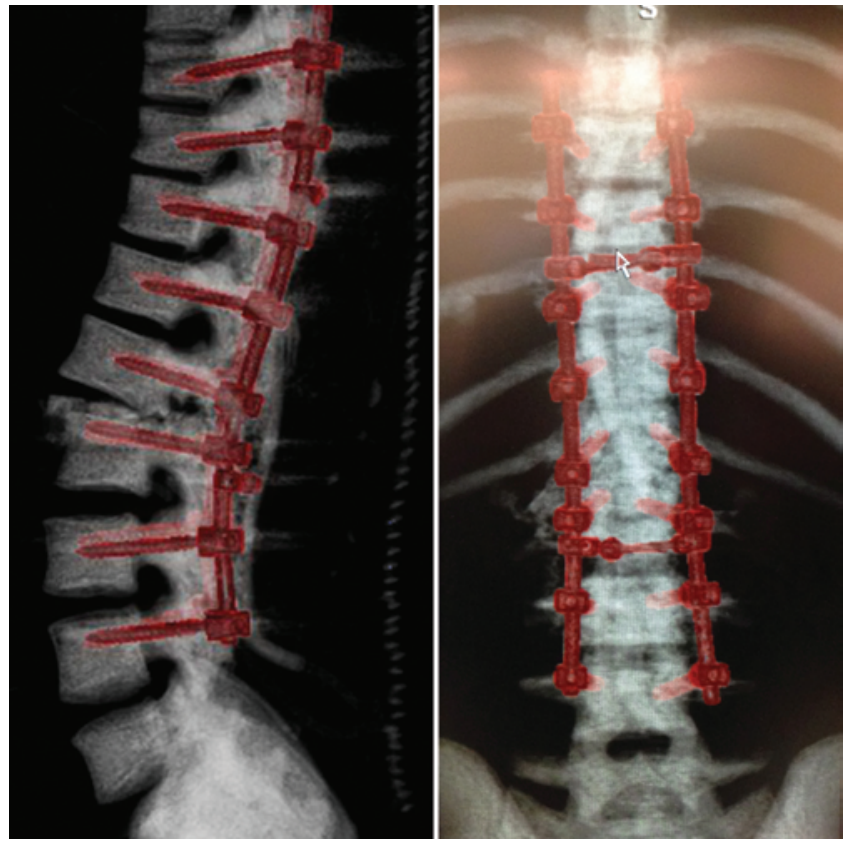

FIG. 3. Postoperative 3D reconstructed CT scans of the thoracolumbar spine. Left: Lateral image demonstrating the T8-L4 instrumentation construct. Right: Coronal image demonstrating the coronal realignment following the instrumented reconstruction. Figure is available in color online only.

above and below the completely resected L-1 vertebral body. This was followed by curettage of the inferior endplate of T-12 and the superior endplate of L-2 in preparation for interbody fusion. Autograft obtained from the L-1 vertebrectomy was then placed into the T12-L2 interspace. This was done to help promote arthrodesis at the T12-L2 interspace. Final rod contouring and placement completed the overall construct, which spanned from T-8 to L-4 (Figs. 2C and 3).

Prior to completing the case, a Valsalva maneuver was performed to evaluate for any evidence of CSF leak; none was seen. A layer of DuraSeal was applied to the sutureligated thecal sac, spanning the laminectomy and fracture defect. The posterolateral surfaces were then decorticated and a combination of allograft and the morselized autograft obtained from the L-1 vertebrectomy were placed from T-8 to L-4 to achieve posterolateral arthrodesis (Fig. 2C).

Intraoperatively, the patient did not experience any injury to the thoracolumbar vasculature. We attribute this to the minimal manipulation that was necessary to realign the spine after completing an L-1 vertebrectomy.

\section{Postoperative Course}

Clinically, the patient had a remarkably uneventful hospital course. Fortunately, he did not experience any of the complications that frequently accompany complete SCI, such as pneumonia, decubitus ulcers, or deep venous thrombosis. One week after the operation, he was discharged to a rehabilitation facility where he learned how to independently transfer to a wheelchair and navigate his
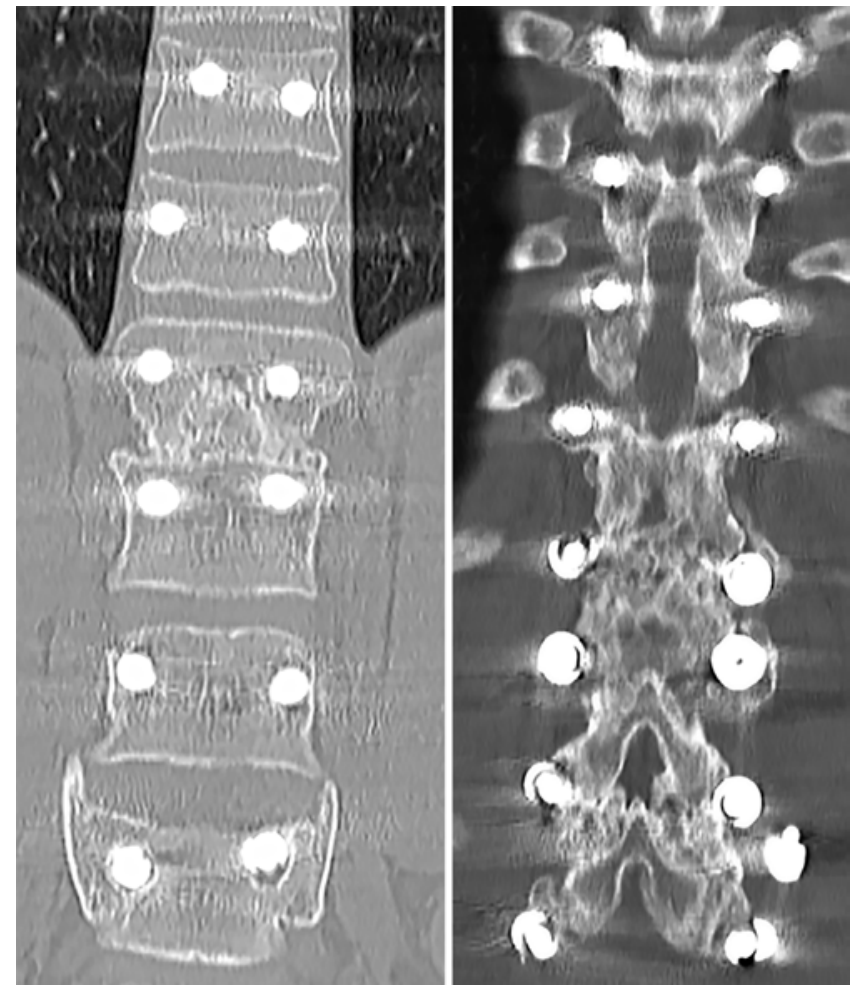

FIG. 4. Coronal CT scans obtained 2 years postoperatively, demonstrating interbody fusion at the level of the spondyloptosis (left) and posterolateral fusion along the instrumented construct (right).

surroundings. He was able to wean off his narcotic pain medication and transition to medicinal marijuana to control long-term chronic pain symptoms. Within months of therapy, he enrolled and attended courses at a local college and regained personal independence by learning to drive a motor vehicle with hand controls.

At the 2-year follow-up, the patient had clinical and radiographic evidence of interbody fusion at T12-L2 (Fig. 4 left) and posteriorly along the entire length of the construct (Fig. 4 right).

\section{Discussion}

Traumatic spondyloptosis of the thoracolumbar junction is a rare occurrence that usually results in complete loss of neurological function below the level of injury. The main goals of surgical intervention include decompressing neural and vascular elements, reducing the fracture to restore sagittal balance, and placing segmental instrumentation to provide stability in an effort to facilitate the patient's return to a new level of normal function. In previously reported cases of thoracolumbar spondyloptosis, fracture reduction was achieved using instrumentation to leverage the spinal column into alignment. ${ }^{1,9,10}$

The force needed to realign the vertebral column is great and has the potential to cause collateral damage. . $^{3,4,7}$ This can include fracture of the instrumented pedicles/ vertebral bodies or injury to critical vascular structures immediately anterior to the spinal column. ${ }^{2,4}$ We offer an alternative correction technique for this type of traumatic 
injury that can be carried out with minimal risk for causing collateral injury to the osseous or vascular structures.

A vertebrectomy was initially performed at L-1 to allow the spinal column to reduce without the application of external forces. Our goal was to achieve realignment and stabilization of the spine in a manner that reduced the risk of injury to surrounding vascular and visceral anatomy. In the setting of a fracture of this magnitude, the possibility of damage to adjacent vital structures is significant. ${ }^{2}$ Attempting to restore normal vertebral column height through massive distraction measures may unnecessarily risk injury to critical vascular structures (distal aorta and inferior vena cava, as well as the iliac arteries and veins).

In a normally aligned spine, performing a vertebrectomy carries a concomitant risk of injuring structures anterior to the spine, such as major vessels, the lungs, or retroperitoneal structures and organs. However, in this setting, these risks are completely eliminated by the location of the vertebral body to be resected resting behind a completely intact spinal column anteriorly. Therefore, the intact inferior aspect of the thoracic spine actually served to protect all structures that would otherwise have been at risk for potential injury during a vertebrectomy if the patient had not experienced a complete spondyloptosis at this level. This unique clinical scenario eliminates many of the potential risks associated with performing a vertebrectomy.

Harvesting the entire L-1 vertebral body for autograft precluded the need to use iliac crest graft, thus reducing the opportunity for well-documented donor site comorbidities, ${ }^{1,6,8}$ although these comorbidities are generally not significant in patients with ASIA Grade A SCI. Furthermore, the volume of autograft obtained from a complete L-1 vertebrectomy far surpasses that which could be obtained from an iliac crest graft alone. Increasing the amount of autograft used for our arthrodesis optimized the patient's potential for successful fusion.

Determining how many levels to include in the construct is based on the type of fracture, severity of the deformity, and integrity of the anterior column structures. ${ }^{3}$ In cases of spondyloptosis the deformity is severe and the anterior column elements are completely destroyed. To ensure that the realigned spine was adequately supported, a long-segment instrumented construct from T-8 to L-4 was performed to maintain sagittal alignment and provide axial stability. Deciding how many levels to include in this type of reconstruction is always a point of contention. Some would argue that instrumentation from T-10 to L-4 would have been adequate, while others would assert that the construct should have been carried from the apex of the thoracic curvature at T-6 to the pelvis. We chose to instrument from T-8 to L-4 to provide an adequate number of fixation points for stability while preventing the intraoperative and postoperative morbidity associated with longer constructs. 3

In this case, all transpedicular instrumentation was placed with the assistance of O-arm navigation, which allowed for efficient and accurate placement. There were no breaches of the pedicles at any of the levels, and none of the screws had to be removed and redirected. Use of intraoperative fluoroscopy or freehand pedicle screw placement would have been acceptable alternatives in this setting.

\section{Conclusions}

Traumatic spondyloptosis at the thoracolumbar junction is a surgical condition that must be addressed with reconstruction of the spinal column with instrumentation techniques. The use of a complete vertebrectomy to assist in realignment offers an additional technique that lessens the risk to surrounding vital structures and decreases instrumentation stress incurred in standard reduction techniques. Reconstruction with local autograft and longsegment instrumentation are viable techniques to provide axial stability and maintain sagittal alignment.

\section{References}

1. Chandrashekhara SH, Kumar A, Gamanagatti S, Kapoor K, Mukund A, Aggarwal D, et al: Unusual traumatic spondyloptosis causing complete transaction of spinal cord. Int Orthop 35:1671-1675, 2011

2. McAfee PC, Werner FW, Glisson RR: A biomechanical analysis of spinal instrumentation systems in thoracolumbar fractures. Comparison of traditional Harrington distraction instrumentation with segmental spinal instrumentation. Spine (Phila Pa 1976) 10:204-217, 1985

3. McLain RF: The biomechanics of long versus short fixation for thoracolumbar spine fractures. Spine (Phila Pa 1976) 31 (11 Suppl):S70-S79, S104, 2006

4. McLain RF, Burkus JK, Benson DR: Segmental instrumentation for thoracic and thoracolumbar fractures: prospective analysis of construct survival and five-year follow-up. Spine J 1:310-323, 2001

5. Norton RP, Milne EL, Kaimrajh DN, Eismont FJ, Latta LL, Williams SK: Biomechanical analysis of four- versus sixscrew constructs for short-segment pedicle screw and rod instrumentation of unstable thoracolumbar fractures. Spine J 14:1734-1739, 2014

6. Park JJ, Hershman SH, Kim YH: Updates in the use of bone grafts in the lumbar spine. Bull Hosp Jt Dis (2013) 71:3948, 2013

7. Parker JW, Lane JR, Karaikovic EE, Gaines RW: Successful short-segment instrumentation and fusion for thoracolumbar spine fractures: a consecutive 41/2-year series. Spine (Phila Pa 1976) 25:1157-1170, 2000

8. Rihn JA, Kirkpatrick K, Albert TJ: Graft options in posterolateral and posterior interbody lumbar fusion. Spine (Phila Pa 1976) 35:1629-1639, 2010

9. Sekhon LHS, Sears W, Lynch JJ: Surgical management of traumatic thoracic spondyloptosis: review of 2 cases. J Clin Neurosci 14:770-775, 2007

10. Yadla S, Lebude B, Tender GC, Sharan AD, Harrop JS, Hilibrand AS, et al: Traumatic spondyloptosis of the thoracolumbar spine. J Neurosurg Spine 9:145-151, 2008

\section{Author Contributions}

Conception and design: Fahim. Acquisition of data: all authors. Analysis and interpretation of data: Fahim, Sandquist. Drafting the article: Sandquist, Paris. Critically revising the article: Fahim, Sandquist. Reviewed submitted version of manuscript: Fahim, Sandquist. Approved the final version of the manuscript on behalf of all authors: Fahim.

\section{Correspondence}

Daniel K. Fahim, Department of Neurosurgery, 3577 W. 13 Mile Rd., Ste. 206, Royal Oak, MI 48073. email: daniel.fahim@ beaumont.edu. 